\title{
Management Strategies for Communited Fractures of Frontal Skull Base: An Institutional Experience
}

\author{
V. Velho ${ }^{1}$ Hrushikesh U. Kharosekar ${ }^{1}$ Jasmeet S. Thukral ${ }^{1}$ Shonali Valsangkar $^{1}$ P. Survashe ${ }^{1}$ \\ ${ }^{1}$ Department of Neurosurgery, Sir Jamshedjee Jeejeebhoy Group of \\ Address for correspondence Dr. Hrushikesh U. Kharosekar, MCh, \\ Department of Neurosurgery, 4th floor, Grant Medical College and Sir \\ Jamshedjee Jeejeebhoy Group of Hospitals, Byculla E, Mumbai \\ Indian J Neurosurg 2015;4:80-84. \\ 400008, Maharashtra, India (e-mail: hkharosekar@gmail.com).
}

\begin{abstract}
Aim The aim of this study is to present our experience in management of complex, communited fractures of the frontal skull base.

Patients and Methods This was a retrospective study performed between January 2000 to January 2013. Data of 1,935 patients with head trauma operated in our department during this period were analyzed. Overall, 210 patients with compound anterior skull base fractures were reviewed. Patients were classified based on radiological features in three groups. Standard neurosurgical protocol was followed for all patients after trauma; reconstruction using titanium mesh, miniplates,

Keywords

- frontal depressed fractures

- cranioplasty

- titanium mesh and silastic graft was done 6 months to 1 year later.

Conclusion Frontal depressed fractures involving the anterior cranial base are complex and require a definitive plan of approach. Reconstruction of bony and dural defects reduces cerebrospinal fluid leak, brain herniation, and infection. Cosmetic reconstruction of the frontal deformity is necessary at a later stage.
\end{abstract}

\section{Introduction}

Fractures within the bony elements of the skull base occur in approximately $25 \%$ of all blunt head injuries. India being a developing country, high speed accidents, and delay in primary care leads to high mortality in these patients. Skull base trauma causes injury to the neurovascular and bony elements of the orbital plate, nasal bones, and anterior cranial base. Most commonly, skull base fractures result from direct trauma either to the frontal and supraorbital regions or to the occiput. Cranial nerve and major arterial and venous injuries are more common in traumatic brain injury accompanied by a skull base fracture.

Frontal basal depressed fractures involving anterior cranial base, occur because of the high velocity injuries. Associated involvement of the sagittal sinus, frontal and ethmoidal sinuses, dural lacerations, contamination,

received

September 24, 2014

accepted

DOI http://dx.doi.org/

$10.1055 / \mathrm{s}-0035-1558836$

ISSN 2277-954X makes management difficult. Burstein et al suggest following classification system based on computed tomographic (CT) scan for anterior skull base fracturestype 1 (central) fracture confined to upper nasoethmoidal complex, central frontal squama, and medial third of superior orbital ramus. Type 2 (unilateral) fracture involves the entire superior rim and upper lateral orbital wall. Type 3 (bilateral) fracture involves fractures of upper nasal ethmoid complex, bilateral supraorbital, and upper lateral orbital, as well as bilateral frontal squama (-Fig. 1).

These fractures require expert and combined efforts in management to prevent major life-threatening catastrophes such as intracranial hematomas, infections, and major cosmetic deformities.

The aim of our study is to present our experience in management of complex frontobasilar fractures and clinical outcome in these patients. ${ }^{1,2}$
License terms of India 

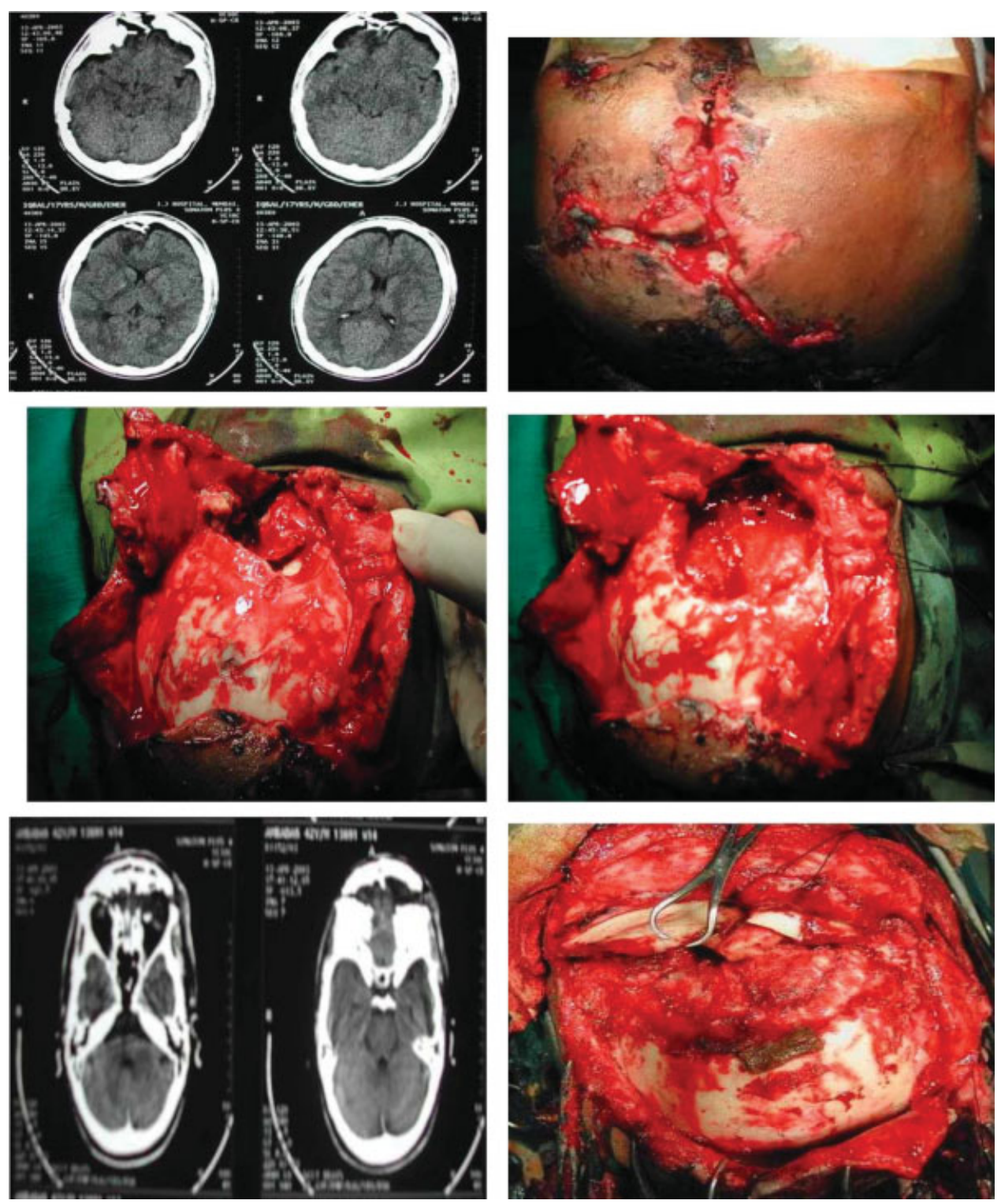

Fig. 1 Two cases of anterior skull base fractures.

\section{Patients and Methods}

Patient data: Retrospective analyses of 1,935 patients with head trauma admitted to our department during the period of January 2001 to December 2012 were reviewed. Overall, 210 patients among these with frontobasilar skull fractures were included in our study. Overall, 137 patients had associated injuries for which they were managed initially and then treated for frontobasilar fractures.

Diagnostic criteria: All patients were initially evaluated in casualty department. Patients were evaluated for history, mode of injury, associated injuries, and relevant history. Radiological evaluation included skull X-ray, CT scan of brain with contrast, and three-dimensional reconstructions. Routine blood investigation, blood grouping, and ultrasound of abdomen were done for every patient as a protocol.
Management protocol: Once the patient as stabilized and managed for associated injuries needing immediate attention patients were considered for prompt neurosurgical management.

Patients with minimally depressed fractures, without any intracranial hematoma or clinical evidence of cerebrospinal fluid (CSF) leak were managed conservatively. Preoperatively dose of higher antibiotics was given. Patients with communited depressed fractures, $>2$-cm depressed segment, significant intracranial hematoma, and contusions were operated immediately. The goal of the surgery was thorough debridement of wound using jet irrigation of hydrogen peroxide and antibiotic saline. Removal of foreign bodies and in-driven bony fragments was done. Any sinus bleed was managed promptly with surgical, gelfoam, and repaired with 6-0 prolene suture. If frontal or ethmoid sinus was involved, it 
was exteriorized with pericranium after removal of mucosa and irrigation with antibiotic solution. Skin loss was covered with rotational flap. In patients with dural tear and expected CSF leak, lumbar drain was kept for minimum period of 72 hour and maximum for 7 days. An attempt was made in every case to on-lay the skull base with pericranium to prevent CSF leak. Orbital rim was reconstructed with titanium miniplates immediately.

Cosmetic deformity was corrected 6 to 12 months later using titanium mesh or methyl methacrylate graft depending on the patient's condition and cosmetic defect.

Evaluation criteria: Patients were evaluated for neurological deficits, convulsions, CSF leak, intracranial infections, wound infection, and cosmetic deformity.

\section{Results}

In our study, maximum patients, 104 , were in 20 to 30 years age group (49.5\%), that is, young adults. Overall, $88 \%$ were male patients. Railway accident was most frequent mode of injury in 114 patients (54.2\%).

Overall, 160 patients (76\%) presented in altered sensorium, 186 (88.5\%) had contaminated wounds. Overall, 32 patients (15\%) had CSF leak at presentation.

On examination, 148 patients (70\%) had Glasgow Coma Scale (GCS) 12-14 at admission. Overall, 28 patients were GCS $<7$ and were intubated immediately and shifted to operation theater.
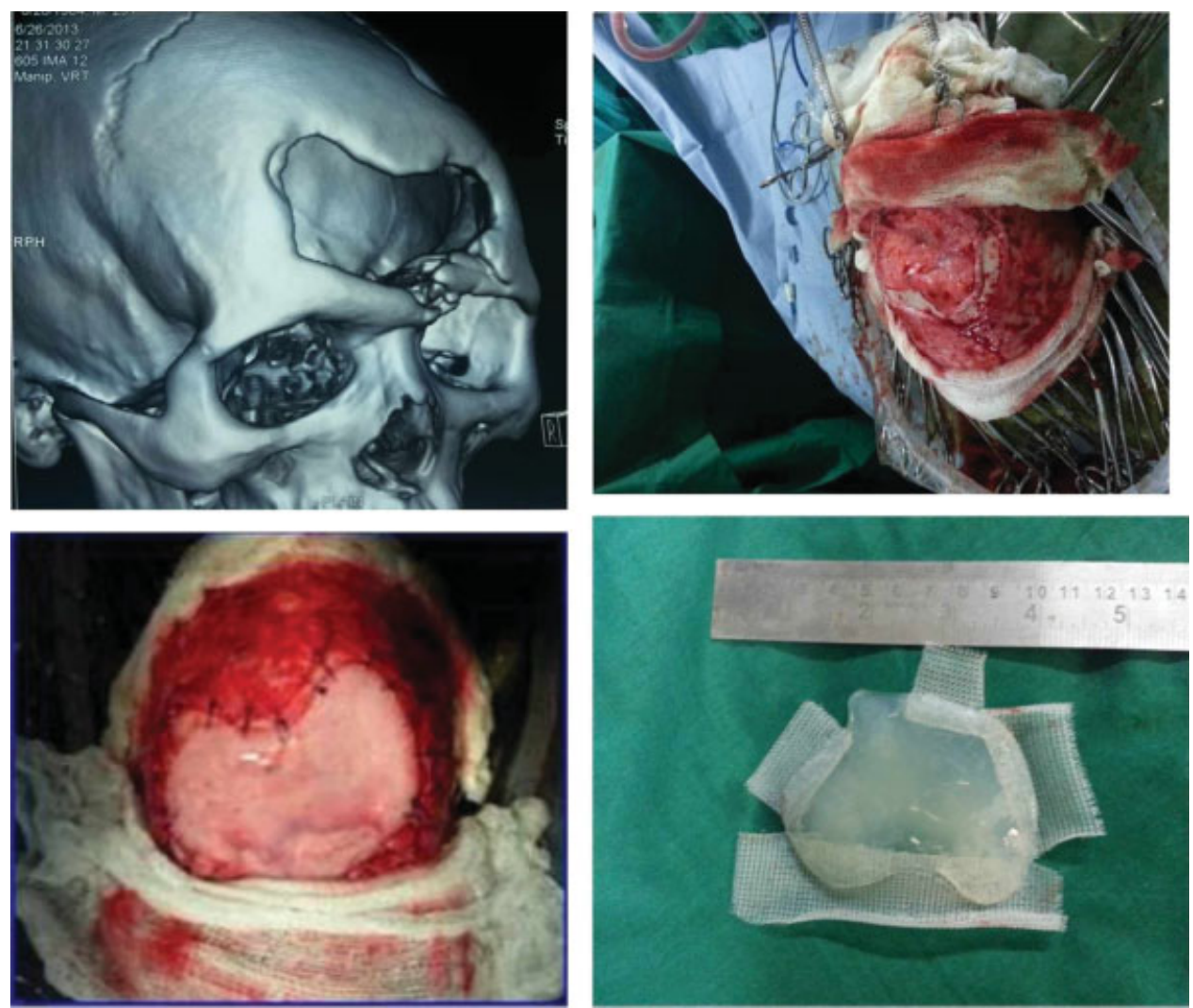

Fig. 2 Cranioplasty using silastic implant.

On radiological examination, 95\% (198) patients had frontal sinus fracture, 110 patients had ethmoid sinus fracture. Other associated findings were orbit fracture (25), extradural hematoma (56), pneumocephalus (52), and contusions (76). Intracranial hematoma was seen in 47 patients, cerebral edema was seen in 109 (\%) patients, right side anterior cruciate ligament (ACA) and middle cerebral artery (MCA) infarct was seen in one patient.

Complication rate was low in our series, most common complication was wound infection seen in 22 patients (11\%) and meningitis was seen in 14 patients (7\%). Overall, 18 patients died postoperatively (8.5\%). Overall, 182 patients recovered well and were back to work at 6-month follow-up.

Cosmetic deformities were corrected later at 6 to 12 months. Overall, 162 patients required reconstructive surgery. Reconstruction was done using methymethacrylate silastic graft in 102 patients and titanium mesh in 62 patients. There was no difference in wound infection and serous collection incidence for both reconstruction but graft rejection as seen in five patients of silastic graft.

\section{Discussion}

The anterior cranial base consists of several segments that vary significantly in rigidity and orientation relative to the horizontal plane and in their proximity to different compartments. Various authors have suggested classification that allows more precise localization and a better understanding of 

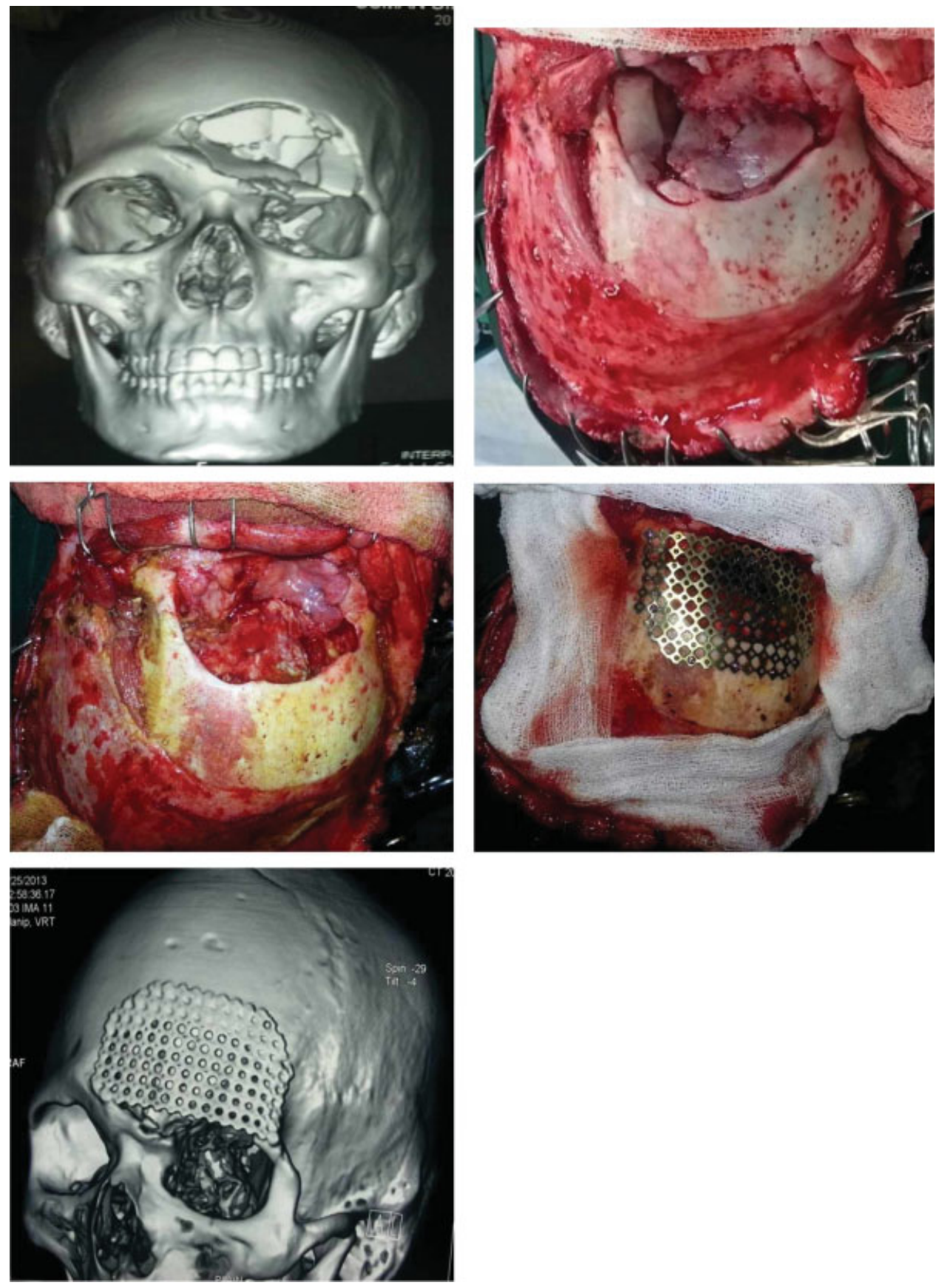

Fig. 3 Cranioplasty using titanium mesh implant.

specific fractures. We adopted the following working classification for management of our patients.

1. Midline frontobasilar fractures (involving central frontal bone, upper nasoethmoid complex, and medial orbital rim): a. With frontal sinus.

b. Without frontal sinus.

2. Unilateral fractures (unilateral frontal bone, orbital rim, and frontal sinus fracture):

a. With posterior wall.

b. Without posterior wall.

3. Bilateral fractures.
4. Complex injuries.

5. Associated vascular injury.

This classification is a CT scan based and helps in categorizing the patients and accurate planning of craniotomies. This classification also helps in delineating the extent of dural damage and parenchymal injury. Although a similar classification has been proposed by Burstein et al, but the main difference is the categorization of patients who are at risk of developing meningitis and in prognosticating the patients who have vascular injuries as this group has poor outcome than other group. 
84 Management Strategies for Communited Fractures of Frontal Skull Base Velho et al.

Simple or minimally depressed fracture $(10 \mathrm{~mm})$ without clinical or radiological evidence of CSF leak were managed conservatively $(n=13)$ with antibiotics and anticonvulsants depending on the extent of brain parenchymal damage.

Any communited or depressed fracture greater than $10 \mathrm{~mm}$ depth was explored after initial stabilization in our series $(n=197)$.

Fractures associated with CSF leak, or presence of significant contusion and significant edema, or involvement of frontal sinus was promptly explored. The key step during surgery was preservation of pericranium, exposure of base to delineate fractures, removal of depressed fragments, thorough debridement of dead and devitalized tissue, generous wash followed by exteriorization of frontal sinus, and followed by brain isolation using water-tight dural closure. Decision regarding bony reconstruction was made depending on the extent of contamination, underlying brain damage and the extent of bony damage and loss. In cases of severely contaminated and comminuted fractures with bone loss thorough debridement followed by reconstruction at a later date yielded acceptable cosmetic outcome.

In presence of severe brain injury that had required decompression, reconstruction was withheld till 3 months, whereas depressed fractures with minimal level of contamination and bone loss were reconstructed immediately.

A majority of patients in our series had frontal sinus injury that was dealt with cranialization or exteriorization. This includes removal of the mucosa of frontal sinus rinsing it with bactericidal solution, packing it with fat followed by covering it with vascularized pericranial graft that was harvested at the beginning of the surgery. We have used fibrin sealant to reinforce the graft as most of our patients had bad contaminated wounds and had presented after a delay of many hours.

Following surgery all the patients were kept in dedicated neurosurgical unit and regularly monitored. Because of close association of frontobasal fractures with orbit, a formal ophthalmology consultation was obtained in every case.

Before reconstruction, all our cases were seen and evaluated simultaneously by a plastic surgeon and the operative decision regarding type of reconstruction was made after a discussion. All cases were investigated with 3D CT cranium and face; Reconstruction was done either by using silastic implant, PMMA, titanium mesh, osteomesh, or a combination of these depending on the region involved, and the financial constraints. In cases where orbital rim was damaged, a new rim was reconstructed using thin titanium plate; whenever available strip of osteomesh was placed and hitched to this plate. The cranial defect was repaired using silastic implant or a titanium mesh (-Figs. 2 and $\mathbf{3}$ ).

\section{References}

1 El Rifaie KM, Taher AA. Frontobasilar Fractures- Guidlines to Management. Egypt J Plas ReconstrSurg 2003;27(2): 249-254

2 Burstein F, Cohen S, Hudgins R, Boydston W. Frontal basilar trauma: classification and treatment. Plast Reconstr Surg 1997; 99(5):1314-1321, discussion 1322-1323 